\title{
THE HYDROGEN CLOUDS OF COMETS
}

\author{
MIKIO SHIMIZU \\ ISAS, Sagamihara \\ Kanagawa 229, Japan
}

\begin{abstract}
In a cometary coma, various hydrogen compounds, especially water, that are photodissociated by solar ultraviolet radiation form a gigantic hydrogen cloud with a radius of several million kilometers around the cometary nucleus. These cometary hydrogen clouds have been observed by a number of spacecraft outside the terrestrial atmosphere and in interplanetary space. For instance, the hydrogen cloud of Comet Halley was a good target for the ultraviolet photometers and spectrometers on board the International Ultraviolet Explorer (IUE), Suisei, Pioneer Venus, DE-1, and sounding rockets. Hydrogen cloud activity could be used as a good measure of cometary activity, but some problems remain to be explored in the future.
\end{abstract}

\section{Introduction}

In 1967 -1968, when the author was one of the visiting fellows (the youngest) of the Joint Institute for Laboratory Astrophysics (JILA), University of Colorado and the National Bureau of Standards, Dr. L. Biermann was another visiting fellow (the eldest). In the JILA seminar, Dr. Biermann gave a lecture on comets, in which he predicted that around a comet existed a gigantic hydrogen cloud formed by photodissociation of water, a possible candidate for the major cometary component suggested by the dirty-ice model of comets by Dr. F. Whipple.

Two years later, the OAO-2 satellite was launched to study celestial bodies in the ultraviolet. Just after this satellite's launching, two big comets, Tago-Sato-Kosaka and Bennett, appeared, and the ultraviolet photometer of OAO-2 found, at the Lyman alpha wavelength $(121.6 \mathrm{~nm})$, the comets' brilliant hydrogen clouds (or comae) extending out to millions of kilometers (Code and Savage, 1972), which is an order of magnitude larger than the extent of the visual cometary comae. This finding gave the first direct evidence supporting the icy conglomerate model of comets, and it also confirmed the presence of the $\mathrm{OH}$ cloud (which had, at that time, been seen only weakly in the ground-based observations). Since then, the hydrogen clouds of comets have been good targets for observations 
by various spacecraft, such as OGO-5, Copernicus, IUE, Pioneer Venus, and Suisei, and also by sounding rockets, Skylab, etc.

Variations in the activity of comets have frequently been discussed by analysis of observations of the hydrogen cloud. This article will concisely review the history and achievements of the Lyman alpha observations.

\section{Formation Processes of the Hydrogen Cloud}

A cometary atmosphere is much more tenuous than a planetary atmosphere: The atomic density at the surface of a fairly large comet is about $10^{12} \mathrm{~cm}^{-3}$, lower by $10^{7}$ than that of the terrestrial atmosphere at the Earth's surface. However, since the cometary density corresponds to that of the planetary ionospheric region, the description of the atmosphere by a fluid dynamic model, similar to the models of the upper atmosphere of a planet, is still possible.

Another difference between a cometary atmosphere and a planetary atmosphere is that the extent of a cometary atmosphere reaches outward some one million kilometers, while that of a planetary atmosphere extends out to only some thousands of kilometers. The big difference comes from the fact that the hydrogen atoms produced by photodissociation of water in the cometary atmosphere have a velocity of about $20 \mathrm{~km} \mathrm{~s}^{-1}$ relative to a nucleus, and these atoms expand almost freely to space until they are photoionized by the solar wind and disappear. On the other hand, the atomic hydrogen in the planetary exosphere is strongly held by planetary gravity and can expand out to merely a few planetary radii.

The density distribution of the cometary hydrogen clouds in the outer coma region discussed above is well-described by the Haser model with one or two scale lengths. However, the scale length fitted to the observations is usually smaller than the one calculated using the photoionization lifetimes (computed from the solar wind flux and measured charged exchange cross-sections) and the estimated $\mathrm{H}$-atom velocity. This is because the Haser model assumes that $\mathrm{H}$-atom ejection is from a point source, while real (isotropic) photodissociation of water occurs in an extended region around the nucleus (Combi and Delsemme, 1980; Festou, 1981).

Furthermore, analysis of the observed profiles of the hydrogen clouds of various comets revealed that some other low-velocity $\mathrm{H}$ components, such as those at $8 \mathrm{~km} \mathrm{~s}^{-1}$ and $4 \mathrm{~km} \mathrm{~s}^{-1}$, are necessary for a good fitting to the observations. The $8 \mathrm{~km} \mathrm{~s}^{-1}$ component can be ascribed to the hydrogen atoms produced from photodissociation of $\mathrm{OH}$, the partner of the photodissociation of water (Brozowski et al., 1978). Various processes for the photodissociation of water are summarized in Table 1.

On the other hand, the $4 \mathrm{~km} \mathrm{~s}^{-1}$ component appears to come not from a molecular process, but rather from a dynamical one, such as collisional thermalization of the $\mathrm{H}$ atom in a transition region between the gas-dynamic and collisionless-exospheric regions. It is usual to use a Monte Carlo computation to represent the complex processes in the transition region (Kitamura et al., 1985). 
Table 1. Photodissociation Processes of Water by Solar Radiation at $1 \mathrm{AU}$

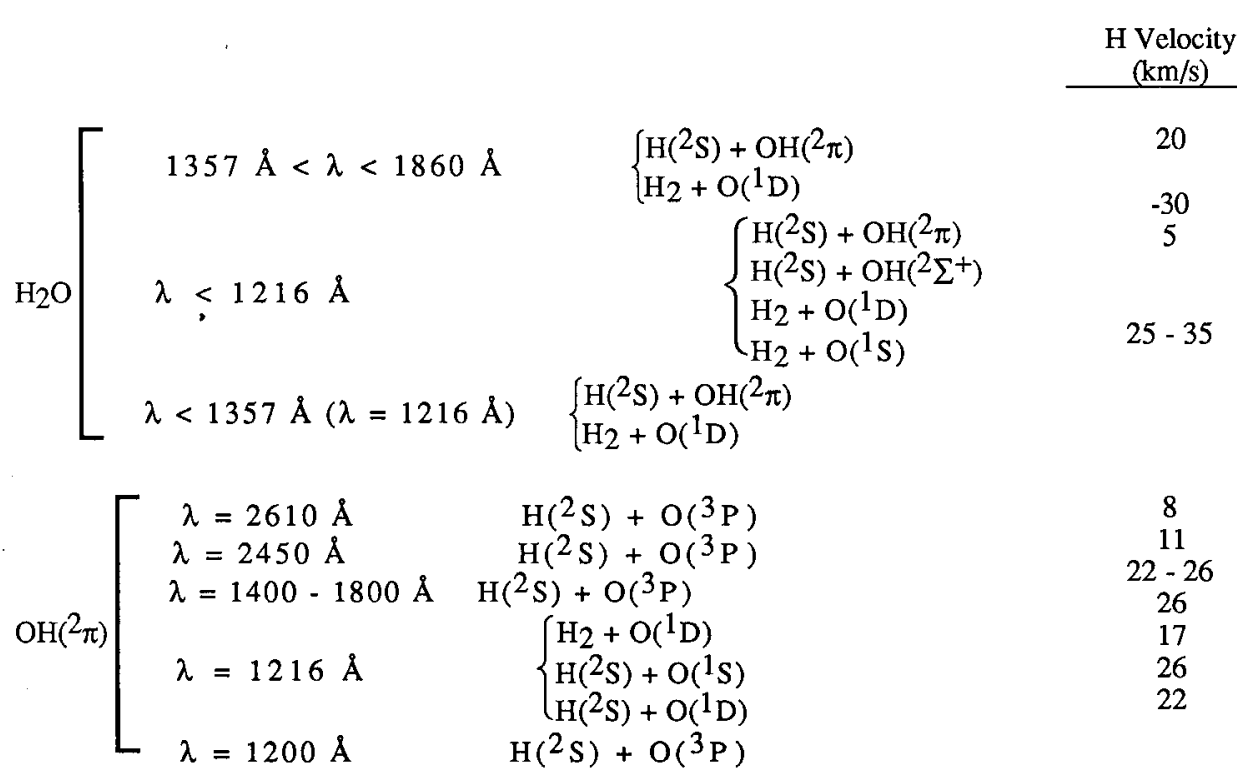

Another complexity in handling the cometary gas is that the cometary coma contains a large number of dust particles, which interact with the gas molecules to decrease the gas velocities. The effect of this on the hydrogen cloud has been computed using a twodimensional axisymmetric model (Kitamura, 1986; for more recent developments, see Combi and Smith, 1988a and 1988b).

Another factor that influences the H-atom distribution is the solar Lyman alpha radiation pressure. The outer isophotes of the hydrogen clouds are severely distorted by this pressure, in particular when the comet approaches the vicinity of the Sun. Furthermore, since the Halley observations, it has become well-established that a comet emits a great deal of its water in the form of jets from localized regions on the nucleus. Variation of the hydrogen cloud associated with these phenomena can also be expected and was actually observed for comets West and Halley, as we shall discuss later.

\section{Hydrogen Cloud Observations in the Pre-Halley Era}

The hydrogen clouds of comets can be observed only from outside of the terrestrial atmosphere, since the hydrogen atoms are seen brilliantly only by resonant scattering of the solar Lyman alpha radiation at $121.6 \mathrm{~nm}$, and this wavelength is completely absorbed by the Earth's atmosphere. Consequently, observations of the hydrogen comae depend 
exclusively on the use of spacecraft. After the first trial observations using OAO-2 and OGO-5 (Keller, 1973a; Keller, 1973b; Keller, 1974; Keller, 1975; Keller, 1976; Keller and Thomas, 1976; Keller and Meier, 1976; Keller and Lillie, 1978), some of the bright new comets such as Kohoutek and West were imaged by rockets (Carruthers et al., 1974; Opal and Carruthers, 1974; Opal et al., 1975), Skylab (Keller et al., 1975; Meier et al., 1976), and Copernicus (Drake et al., 1976; Festou et al., 1983).

Most of the observed isophotes of these comets were analyzed in a single scattering approximation, using the syndyne model to take the solar radiation pressure into account. However, a multiple scattering approximation is necessary for exact analysis of the optically thick region of the hydrogen clouds. The complexity of the radiative transfer technique has restricted the application of this approximation to only one cometary case (Keller, 1973a and 1973b).

Two or more velocity components are required for interpretation of some observations of the hydrogen clouds. The first direct evidence for the two-step photodissociation model ( $\mathrm{H}$ from $\mathrm{H}_{2} \mathrm{O}$ and $\mathrm{OH}$ ) was obtained when the Copernicus satellite revealed a variation in the line width of the Lyman alpha emission with cometocentric distance in the case of Comet Kobayashi-Berger-Milon: The broad emission profile near the center of the coma was explained in terms of the high-velocity component, while the narrower profile observed far from the nucleus required a lower-velocity component (Festou et al., 1979).

As the production mechanism became clear, the hydrogen cloud observations were frequently used to derive the water production rate dependence on heliocentric distance (Feldman et al., 1980; Weaver et al., 1981a; Weaver et al., 1981b; Feldman, 1982; Festou et al., 1983; Festou, 1986; Festou and Feldman, 1987). In the cases of comets Bennett, Tago-Sato-Kosaka, and West, an $\mathrm{R}^{-2}$ variation was a good fit, while an $\mathrm{R}^{-3.7}$ law was a better description for comets Bradfield (1979X), Encke, Austin, and Crommelin. It was suggested that a dusty comet does not follow a simple sublimation picture, because of a thermally positive feedback interaction of the dust particles with the cometary surface (Weissman and Kieffer, 1979).

It was also found that the ratios of the production rates of various atomic constituents, such as $\mathrm{H}, \mathrm{C}$, and $\mathrm{O}$, vary between comets. If $\mathrm{H}$ comes from $\mathrm{H}_{2} \mathrm{O}$ and $\mathrm{C}$ comes from $\mathrm{CO}$, this variation is natural, since the $\mathrm{CO}$ abundance is known to vary between comets (Weaver et al., 1981a; Weaver et al., 1981b; Wallis and Carey, 1985). However, $\mathrm{CO}$ may not necessarily be the parent molecule itself, and its production directly from the $\mathrm{CHON}$ particles has frequently been advocated. Similarly, $\mathrm{H}$ could be formed from the $\mathrm{CHON}$ particles. Interestingly enough, the February 24 observations of Halley gave an $\mathrm{H} / \mathrm{O}$ ratio of $>3$, perhaps indicating that not all the hydrogen is produced from water (McCoy et al., 1986).

The brightness contours of Comet West could not be explained by the syndyne model with two velocity components. An outburst that accompanied the splitting of this comet's nucleus several days before the observation appears to be the cause of this discrepancy (Keller and Meier, 1980). Since the $\mathrm{H}$ atoms produced by photodissociation of water expand to the distance of about two million kilometers in a day, the hydrogen distribution of the coma is obtained by superposing the atomic density of $\mathrm{H}$ ejected from various points 
of the cometary orbit over a period of a few days. The extent of the cometary isophotes is seriously changed by the outburst phenomena. On the other hand, when the water production rate is a slowly varying function of heliocentric distance, as in the cases of fresh comets, a quasi-stationary treatment of the hydrogen cloud would be a good approximation.

\section{Lyman Alpha Observations of Comet Halley}

The recent apparition of Comet Halley, in 1986, was a good opportunity for exploring a comet in Lyman alpha wavelengths as well as in other wavelengths. Quite new types of hydrogen coma observations were carried out on this occasion: Suisei passed through the interior of the comet's hydrogen cloud, obtaining the first observations of the resulting variation of the 121.6-nm radiation (Kaneda et al., 1986c). Pioneer Venus measured the Halley hydrogen cloud from orbit around Venus (Stewart, 1987). From Earth orbit, IUE observed the cloud almost continuously from September 1985 to July 1986 (Feldman et al., 1987; Festou, 1988). Several Earth-bound observations, such as those by the DE-1 and sounding rockets, were also attempted.

Suisei first detected the hydrogen cloud of Comet Halley on November 28, 1986. During the three weeks of observation time following that date, the spacecraft found a time variation (or breathing) of the Lyman alpha intensity with a period of 2.2 days (Kaneda et al., 1986b). It has been suggested that the breathing was caused by outbursts (jets) from nuclear surface features that were activated when rotation of the nucleus brought them into positions where they were illuminated by the Sun. Before Suisei's observations, the repetition of brighter and darker phases in visible light was reported, but the periods obtained were quite scattered and were a matter of debate with no firm conclusion (e.g., West and Pederson, 1984). The shape of the coma dust features suggested a rotation period of 1.3 to 2.2 days or perhaps a bit longer (Larson and Sekanina, 1985).

As is well-known, however, another period, one of 7.3 days, was reported in the visible-light photometry after perihelion (Millis and Schleider, 1986), and the Pioneer Venus spacecraft obtained a time variation with a good fit to this new value. Some reports of observations made at various wavelengths agreed with this new period, and others disagreed. A possible natural interpretation of this brightness variation is a 2.2-day rotation of the nucleus, with a 7.3-day nutation. The change in what surface region on the nucleus was lighted by the Sun before and after perihelion caused a different pattern of outbursts and may have highlighted the nutational motion (Weissman, 1987).

The sunlight falling upon the nucleus region responsible for the outbursts with a 2.2-day period of repetition might be much reduced after perihelion. A different nucleus region, one in which the radiation upon it was more controlled by the 7.3-day nutation effect, might then become the dominant source of outbursts. Indeed, the 2.2-day variation observed post-perihelion by Suisei (Kaneda et al., 1986c) was weak and was seen only in the immediate vicinity of the nucleus above the stronger and more uniform background, which might exhibit a 7.3-day variation. 
It should be noted that a dynamically important parameter of a rotating body is the angular momentum and that Halley's nutation contributes negligibly to this physical parameter.

The IUE observations of Comet Halley covered heliocentric distances of less than $2.2 \mathrm{AU}$. At $1 \mathrm{AU}$, the IUE's aperture sampled a region of 5,000-km radius about the nucleus, where the hydrogen column was optically thick. The Lyman alpha intensity varied nearly as $\mathrm{r}^{-2}$, where $\mathrm{r}$ is the cometocentric distance, as expected (P.D. Feldman, in this volume).

During Suisei's encounter phase, the spacecraft used a photometry mode in which the image data were compressed into a histogram of the light level distribution in order to reduce the memory requirements for each image from 18,666 bytes to 512 bytes. The field of view for the image was in the direction of the constellation of Leo. Superimposed upon a smooth intensity variation obeying an $\mathrm{r}^{-1}$ law, some fine structure was seen. These clumps may correlate with the outburst phenomena described earlier (Kaneda et al., 1986a; Kaneda et al., 1986b; Shimizu, 1987).

Dependence of Comet Halley's water production rate on the heliocentric distance has been measured not only by the Lyman alpha observations, but also by many other ground-based observations and spacecraft. Although the results of the various measurements were slightly different, the qualitative tendencies were generally in agreement with each other: The rate after perihelion was higher than that before perihelion, by a factor of 2 to 3, which may be due to the strong solar heating of the cometary nucleus in the neighborhood of perihelion. The rate at $1 \mathrm{AU}$ before perihelion was about $4 \times 10^{29} \mathrm{~mol} \mathrm{~s}^{-1}$, and that near the time of the Halley armada encounter was almost $1 \times 10^{30} \mathrm{~mol} \mathrm{~s}^{-1}$. In comparison, the rates obtained by the hydrogen Lyman alpha observations of the sounding rocket (McCoy et al., 1986) and the Pioneer Venus spacecraft (Stewart et al., 1987) were $7 \times 10^{29} \mathrm{~mol} \mathrm{~s}^{-1}$ on March 13 and 1.4 to $1.6 \times 10^{30} \mathrm{~mol} \mathrm{~s}^{-1}$ at the VEGA encounter time, respectively.

These values are all a little higher than was expected before the apparition (Divine et al., 1986). We should be careful to note that, because of the possible contribution of other sources in the production of $\mathrm{H}$ atoms, the hydrogen observations give an upper limit in the determination of water production.

\section{Remaining Problems}

The main formation process of cometary hydrogen clouds is rather well identified as the two-step photodissociation of water. However, other sources, such as $\mathrm{CH}$ and $\mathrm{HCHO}$ in the CHON particles, might contribute to the production of $\mathrm{H}$ atoms (Shimizu, 1986), since these particles have very large photodissociation cross-sections and the total mass of dust particles appears to be much larger than that of gas molecules. Variations over many comets of the ratios of $\mathrm{H}, \mathrm{C}$, and $\mathrm{O}$ also suggest this possibility. The fact that the water production rate dependency on the heliocentric distance varies between comets- 
in particular, between dusty and nondusty comets-shows the complexity of the gas-dust feedback interaction.

Interpretation of the hydrogen cloud in terms of a single scattering model with a syndyne approximation appears to be too simple. A nonstationary model with a multiple scattering treatment might be essential for the description of active comets such as Halley and West. The hydrogen cloud appears to be a simple phenomenon, but it may still contain unexpected complexities to be explored in detail.

\section{References}

Brozowski, J., et al. (1978). "Precision Estimates of the Predissociation Rates of the $\mathrm{OH}$ A State $\left(2 \Sigma^{+}\right), "$ Phys. Scripta, 17, 507-511.

Carruthers, G.R., et al. (1974). "Lyman Alpha Imaginary of Comet Kohoutek," Icarus, 23, 526-537.

Code, A.D., and Savage, D. (1972). "OAO: Review of Scientific Results," Science, 177, 213-221.

Combi, M.R., and Delsemme, A.H. (1980). "Neutral Cometary Atmosphere I. An Average Random Walk Model for Photodissociation in Comets," Ap. J., 237, 633-640.

Combi, M.R., and Smith, W.H. (1988a). "Monte Carlo Particle-Trajectory Models for Neutral Cometary Gases I. Models and Equations," Ap. J., 327, 1026-1043.

Combi, M.R., and Smith, W.H. (1988b). "Monte Carlo Particle-Trajectory Models for Neutral Cometary Gases II. The Spectral Morphology of the Lyman Alpha Coma," Ap. J., 327, 1045-1059.

Divine, N., et al. (1986). "The Comet Halley Dust and Gas Environments," Space Sci. Rev., 3, 1-104.

Drake, J.F., et al. (1976). "Lyman Alpha Observations of Comet Kohoutek," Ap. J., 209, 302-311.

Feldman, P.D. (1982). "Ultraviolet Spectroscopy of Comae," in L.L. Wilkening (ed.), Comets, The University of Arizona Press, Tucson, pp. 461-479.

Feldman, P.D., et al. (1980). "IUE Observation of the UV Spectrum of Comet Bradfield," Nature, 286, 132-135.

Feldman, P.D., et al. (1987). "IUE Observation of P/Halley," Astron. Astrophys., 187, $325-328$.

Festou, M.C. (1981). "The Density Distribution of Neutral Compounds in Cometary Atmospheres I. Models and Equations," Astron. Astrophys., 95, 69-79.

Festou, M.C. (1986). "Comets," ESA SP-263, pp. 3-9.

Festou, M.C. (1988). "Variation of the Gaseous Output of the Nucleus of Comet P/Halley," in J. Mason and P. Moore (eds.), Comet Halley Worldwide Investigations, Results, and Interpretation, in press.

Festou, M.C., and Feldman, P.D. (1987). "Comets," in Y. Kondo (ed.), Scientific Accomplishments of the IUE, D. Reidel Publ. Comp., Dordrecht, pp. 101-118. 
Festou, M., et al. (1979). "Lyman Alpha Observations of Comet Kobayashi-Berger-Milon (1975 IX) with Copernicus," Ap. J., 232, 318-328.

Festou, M.C., et al. (1983). "IUE Observation of Comet P/Crommelin," Astron. Astrophys., 152, 170-172.

Festou, M.C., et al. (1983). "Lyman Alpha Observations of Comet West and P/d'Arrest with Copernicus," Ap. J., 265, 925-932.

Kaneda, E., et al. (1986a). "Activity of Comet Halley Observed in the Ultraviolet," Geophys. Res. Let., 13, 833-836.

Kaneda, E., et al. (1986b). "Strong Breathing of the Hydrogen Coma of Comet Halley," Nature, 320, 140-141.

Kaneda, E., et al. (1986c). "Observation of Comet Halley by the Ultraviolet Imager of Suisei," Nature, 321, 297-299.

Keller, H.U. (1973a). "Lyman Alpha Radiation in the Hydrogen Atmospheres of Comets: A Model with Multiple Scattering," Astron. Astrophys., 23, 269-280.

Keller, H.U. (1973b). "Hydrogen Production Rates of Comet Bennett in the First Half of April, 1970," Astron. Astrophys., 27, 51-57.

Keller, H.U. (1974). "The Scale Length of $\mathrm{OH}$ and the Production Rates of $\mathrm{H}$ and $\mathrm{OH}$ in Comet Bennett," Astron. Astrophys., 34, 187-196.

Keller, H.U. (1975). "A Cometary Hydrogen Model: Comparison with OGO-5 Measurements of Comet Bennett," Astron. Astrophys., 39, 7-19.

Keller, H.U. (1976). "The Interpretations of Ultraviolet Observations of Comets," Space Sci. Rev., 18, 641-684.

Keller, H.U., and Lillie, C.F. (1978). "Hydrogen and Hydroxyl Production Rates of Comet Tago-Sato-Kosaka," Astron. Astrophys., 62, 143-147.

Keller, H.U., and Meier, R.R. (1976). "A Cometary Hydrogen Model for Arbitrary Observational Geometry," Astron. Astrophys., 52, 273-281.

Keller, H.U., and Meier, R.R. (1980). "On the Lyman Alpha Isophotes of Comet West," Astron. Astrophys., 81, 210-214.

Keller, H.U., and Thomas, G.E. ( 1976). "A High Velocity Component of Atomic Hydrogen in Comet Bennett," Proc. IAU Collog. No. 25, 316-321.

Keller, H.U., et al. (1975). "High Resolution Lyman Alpha Observations of Comet Kohoutek Near Perihelion," Astron. Astrophys., 38, 413-416.

Kitamura, Y. (1986). "Axisymmetric Dusty Gas Jet in the Inner Coma of a Comet," Icarus, 66, 245-257.

Kitamura, Y., et al. (1985). "A Model for the Hydrogen Coma of a Comet," Icarus, 61, 278-295.

Larson, S.M., and Sekanina, Z. (1985). "Coma Morphology and Dust-Emission Pattern of Periodic Comet Halley. III. Additional High Resolution Images Taken in 1910," Astron. J., 90, 823-826.

McCoy, R.P., et al. (1986). "Far-Ultraviolet Spectral Images of Comet Halley from Sounding Rockets," Nature, 324, 439-441.

Meier, R.R., et al. (1976). "Hydrogen Production Rate from Lyman Alpha Images of Comet Kohoutek," Astron. Astrophys., 52, 283-290. 
Millis, R.L., and Schleider, D.G. (1986). "Rotational Period of Comet Halley," Nature, 324, 646-649.

Opal, C.B., and Carruthers, G.R. (1975). "Lyman Alpha Observation of Comet West," Icarus, 31, 503-509.

Opal, C.B., et al. (1974). "Comet Kohoutek: Ultraviolet Images and Spectrograms," Science, $185,702-705$.

Shimizu, M. (1986). "Hydrogen Coma of Comet Halley," Adv. Space Res., 5, 73-81.

Shimizu, M. (1987). "Halley's Environments Observed by the Japanese Suisei Spacecraft," ESA SP-278, 229-232.

Stewart, A.I.F. (1987). "Pioneer Venus Measurement of H, O, and C Production in Comet P/Halley Near Perihelion," Astron. Astrophys., 187, 369-374.

Wallis, M.K., and Carey, W.C. (1985). "Observation of Comet Crommelin-V: Anomalous Hydrogen Source," Month. Nat. R. Astr. Soc., 217, 673-678.

Weaver, H.A., et al. (1981a). "IUE Observations of Faint Comets," Icarus, 47, 449-463.

Weaver, H.A., et al. (1981b). "Water Production Models for Comet Bradfield," Ap. J., 251, 809-815.

Weissman, P.R. (1987). "Post-Perihelion Brightening of Comet P/Halley: Springtime for Halley," Astron. Astrophys., 187, 873-878.

Weissman, P.R., and Kieffer, H.H. (1979). "Thermal Modeling of Cometary Nuclei," Icarus, 47, 302-311.

West, R.M., and Pederson, H. (1984). "Variability of P/Halley," Astron. Astrophys., 138, L9-L10. 